\title{
Exopolysaccharides production by Mesorhizobium loti: effect of carbon source and $\mathrm{pH}$
}

\author{
Rutilene Jacondino Roll ${ }^{1}$, Jéssica Dutra Vieira ${ }^{1}$, João Vitor Sales Rosa ${ }^{1}$, Renata \\ Machado Castro ${ }^{1}$, Rafael Hencke Tresbach ${ }^{2}$, Valdir Marcos Stefenon ${ }^{1,2}$ \\ ${ }^{1}$ Núcleo de Microbiologia Aplicada à Biorremediação - NuMAB, Universidade Federal do Pampa, \\ Campus São Gabriel, São Gabriel, RS, Brazil \\ ${ }^{2}$ Programa de Pós Graduação em Ciências Biológicas, Universidade Federal do Pampa, Campus São Gabriel, \\ São Gabriel, RS, Brazil \\ valdirstefenon@unipampa.edu.br
}

\begin{abstract}
Interest in culturing microorganisms for production of valuable polysaccharides has greatly increased and attracted worldwide attention due to their novel and exclusive physical properties. We report the production of exopolysaccharides (EPSs) by two strains of Mesorhizobium loti (SEMIA806 and SEMIA816) under two different $\mathrm{pH}$ conditions and using three different sources of carbon. From 0.105 to $0.599 \mathrm{~g}$. $\mathrm{L}^{-1}$ of EPS was produced by bacteria growing in medium using dextrose as carbon source and from 0.332 to 2.04 g.L. $\mathrm{L}^{-1}$ of EPS when the sucrose was the carbon source employed. The use of mannitol as carbon source resulted in the lower rate of EPS production, not exceeding 0.001 g.L-1 . In both, $\mathrm{pH} 5.5$ and pH 7.0, the amount of EPS produced was higher for SEMIA816 than for SEMIA806. Both strains produced higher amount of EPSs at pH 5.5 and using sucrose as carbon source. Since none of the species of this genus have yet been shown to be pathogenic M. loti SEMIA806 and SEMIA816 can be included among the potential sources of promising biopolymers using stressful growing conditions.
\end{abstract}

Keywords: Biopolymers, EPSs, medium acidity, microbial stress, rhizobia

\section{INTRODUCTION}

Several microorganisms produce extracellular polysaccharides (biopolymers), which can be found attached to the cell surface or extracted from the fermentation medium. In general, bacteria use these microbial biopolymers as storage materials in response to particular environmental stresses (Sanchez Garcia et al. 2010, Dertli et al. 2015). Numerous studies have shown that some extracellular polysaccharides (exopolysaccharides) produced by Rhizobium species constitute a macromolecular interface between the bacterial cell and its environment, promoting a complex series of interactions leading to the establishment of an effective Rhizobium-legume symbiosis (Hotter and Scott 1991, Bomfetti et al. 2011, Tatsukami and Ueda 2016). The exopolysaccharides (EPSs) produced by rhizobia are highly diverse, varying in the type of sugars and their linkage in the single subunit, repeat unit size and polymerization degree, as well as non-carbohydrate decoration. In addition, such EPSs are mostly species or strain-specific heteropolysaccharides (Bomfetti et al. 2011).

Exopolysaccharides have rapidly emerged as an industrially important source of polymeric materials, which are gradually becoming economically competitive (Singh and Saini 2012). Therefore, interest in culturing microorganisms for production of valuable polysaccharides has greatly increased and attracted worldwide attention due to their novel and unique physical properties as bioadhesives, bioflocculants, biosorbents, gelling agents, probiotics, stabilizers, and thickeners. Such properties make microbial EPSs suitable for numerous commercial applications in the bionanotechnology, food, pharmaceutical, cosmetics, petroleum, civil construction, and environmental sectors (Bomfetti et al. 2011, Castellane et al. 2015). 
Despite the absence of studies on the commercial production of gum by rhizobia, they can be considered highly promising unexplored sources of microbial EPSs for industrial applications (Castellane et al. 2015). High-level EPSs producing microbial systems have gain escalating industrial importance due to the very high production costs of microbial EPSs production. So, the search of innovative culture conditions aiming to control and improve microbial productivity of EPSs has gained significance in industrial and medical biotechnology (Ates 2015). In this note we report the production of EPSs by strains SEMIA806 and SEMIA816 of Mesorhizobium loti (former Rhizobium loti, Jarvis et al. 1997), in two different pH conditions and using three different sources of carbon in the culture medium, aiming to contribute to the discussion of the subject optimization of EPSs production with possible commercial interest.

\section{Material AND Methods}

Mesorhizobium lotistrains SEMIA806 and SEMIA816 were kindly provided by the State Foundation of Agricultural Research of the Rio Grande do Sul (FEPAGRO), São Gabriel, Brazil. The M. loti isolates were firstly cultured in Petri plates with solid YM medium (1.0 g.L $\mathrm{L}^{-1}$ yeast extract, 0.5 g.L $\mathrm{L}^{-1} \mathrm{~K}_{2} \mathrm{HPO}_{4}, 0.2$ g.L.-1 $\mathrm{MgSO}_{4}, 0.1$ g.L $\mathrm{L}^{-1} \mathrm{NaCl}, 1.0$ g. $\mathrm{L}^{-1} \mathrm{CaCO}_{3}$ and 10 g.L.- of D-mannitol). The YM medium was solidified with bacteriological agar $\left(15 \mathrm{~g}^{-1} \mathrm{~L}^{-1}\right)$ and sterilized at $1.2 \mathrm{~kg} \cdot \mathrm{cm}^{-1}$ at $121^{\circ} \mathrm{C}$ for $15 \mathrm{~min}$ before inoculation. Plates were maintained during 16 hours at room temperature within a sterilized environment to assure the absence of bacterial or fungal contamination before inoculation with $M$. loti. Actively growing $M$. loti colonies were isolated from these cultures and employed in the analysis of bacteria growth and EPSs production in liquid medium.

To verify the effect of different sources of carbon and medium acidity in bacterial growth and EPS production, single $M$. loti colonies grown in the solid medium were cultured in liquid medium containing 1.0 g. $\mathrm{L}^{-1}$ yeast extract, 0.5 g.L $\mathrm{L}^{-1} \mathrm{~K}_{2} \mathrm{HPO}_{4}, 0.2 \mathrm{~g} . \mathrm{L}^{-1} \mathrm{MgSO}_{4}, 0.1 \mathrm{~g} . \mathrm{L}^{-1} \mathrm{NaCl}, 1.0 \mathrm{~g} . \mathrm{L}^{-1} \mathrm{CaCO}_{3}$ and $10 \mathrm{~g} . \mathrm{L}^{-1}$ of carbon source, i.e D-mannitol (YMan medium), sucrose (YSuc medium) or dextrose (YDex medium). The $\mathrm{pH}$ of the culture media was adjusted to two different values: 5.5 and 7.0, in order to verify the effect of $\mathrm{pH}$ of the growth medium. Before bacteria inoculation, $50 \mathrm{~mL}$ of the medium was dispensed in $250 \mathrm{~mL}$ Erlenmeyer flasks and sterilized at $1.2 \mathrm{~kg}^{-\mathrm{cm}^{-1}}$ at $121^{\circ} \mathrm{C}$ for $15 \mathrm{~min}$ (Figure $1 \mathrm{~A}$ ). Bacteria were grown during seven days at $28^{\circ} \mathrm{C}$, without shaking.

The EPS production was evaluated after seven days of culturing. For the extraction of the EPSs, $50 \mathrm{~mL}$ of the medium was centrifuged in $50 \mathrm{~mL}$ conical centrifuge tubes at $6000 \mathrm{RFC}$ in a centrifuge Rotina 420R (Hettich $\mathrm{GmbH} \& \mathrm{Co}$. KG, Tuttlingen, Germany) during $30 \mathrm{~min}$ at $4^{\circ} \mathrm{C}$ and the EPSs were precipitate with 2 volumes of ethanol $96 \%$. EPSs were dried at $55^{\circ} \mathrm{C}$ until reaching constant weight. The mass was determined using a precision balance M214A (Bel Engineering, Piracicaba, Brazil) (Figure 1B).

All experiments were performed in triplicate and the inoculation steps were performed within a sterilized airflow chamber Model A10 (Pachane Ltda., Piracicaba, Brazil). The difference of the means for comparing the effect of $\mathrm{pH}$, carbon source and strain in EPS production was determined using the Mann-Whitney test.

\section{RESULTS AND Discussion}

From 0.105 to 0.599 g.L $\mathrm{L}^{-1}$ of EPS was produced by bacteria growing in medium using dextrose as carbon source. This amount ranged from 0.332 to $2.04 \mathrm{~g} . \mathrm{L}^{-1}$ of EPS when the sucrose was the carbon source employed. The use of mannitol as carbon source resulted in the lower rate of EPS production, not exceeding $0.001 \mathrm{~g} . \mathrm{L}^{-1}$ of EPS.

The amount of EPS produced by M. loti SEMIA806 was not significantly affected ( $p>0.38$ ) by the medium acidity (pH $5.5 \mathrm{vs} \mathrm{pH} \mathrm{7.0),} \mathrm{independent} \mathrm{of} \mathrm{the} \mathrm{employed} \mathrm{carbon} \mathrm{source.} \mathrm{However,} \mathrm{in} \mathrm{pH} \mathrm{5.5,} \mathrm{1.8-fold} \mathrm{higher}$ production of EPS was observed for the culture using sucrose and 2.5-fold for the culture using dextrose (Figure 2). For the strain $M$. loti SEMIA816, significant difference $(\mathrm{p}=0.05)$ between $\mathrm{pH}$ conditions was observed for the EPS production in the YSuc and YDex medium (Figure 2). In the medium YMan, no difference was observed when comparing the $\mathrm{pH}$ conditions, for none of the strains. 
When the strains were compared, $M$. loti SEMIA806 revealed significantly higher $(\mathrm{p}=0.005)$ production of EPS under neutral $\mathrm{pH}$ with dextrose and sucrose as carbon source, while M. loti SEMIA816 produced higher amount $(p=0.05)$ with sucrose as carbon source under both, neutral and acid conditions. No significant difference ( $p$ $=0.88$ ) between strains was observed for pH 5.5 in the YDex medium (Figure 2). No difference was observed between strains for the medium YMan in none of the $\mathrm{pH}$ conditions.

Both strains employed in this study have been recommended for the inoculation production for leguminous species expected to grow in acid soils, such as Lotus corniculatus L. (Oliveira et al. 2007, Martins et al. 2011). However, it is know that some species of genus Rhizobium are moderately acid tolerant, while others are very sensitive to acidity (Wood et al. 1988, Brockwell et al. 1991, Tiwari et al. 1992). Similarly, different strains of the same species may vary widely in their $\mathrm{pH}$ tolerance (Correa and Barniex 1997, Bomfetti et al. 2011). The results of our study corroborate previous studies about the tolerance to acid conditions of $M$. loti (e.g. Wood et al. 1988) and also the different response of strains for $\mathrm{pH}$ conditions. Both strains produced higher amount of EPS under acidic conditions when sucrose and dextrose were used as carbon source. These results reflect the nature of bacteria producing EPS when under stressful environments. Using glycerol as carbon source, Barberi et al. (2004) reported higher production of EPS for Bradyrhizobium elkanii (strain SEMIA5019) under pH 5.5, in comparison to $\mathrm{pH} 6.0$ and 6.8. Clearly, M. loti strain SEMIA816 produced much high amount of EPS when grown in YSuc and under pH 5.5, a more stressful condition. Since mannitol is the most recommended source of carbon for soil bacteria, no stressful condition was created, resulting in such very low EPS production for both strains and in both pH values. Studies on the growth of M. lotti SEMIA806 and Bradyrhizobium japonicum SEMIA5080 performed using different sources of carbon and different $\mathrm{pH}$ conditions for in vitro culture corroborate this inference. Vieira et al. (2016) observed lower bacterial growth due to more stressful conditions in cultures with sucrose and dextrose under $\mathrm{pH} 5.5$, in comparison to mannitol and $\mathrm{pH} 7.0$, respectively.

In conclusion, both strains of M. loti tested in this study produced higher amount of EPSs at pH 5.5 and using sucrose as carbon source. Since none of the species of the genus Rhizobium have yet been shown to be pathogenic (Castellane et al. 2015), M. loti SEMIA806 and SEMIA816 can be included among the potential sources of promising biopolymers. However, the characterization of the EPSs' compounds is needed since the culture conditions, the media composition, and even different bacterial strains may produce EPSs with distinct chemical composition (Fraysse et al. 2003, Bomffetti et al. 2011, Donot et al. 2012, Castellane et al. 2015).
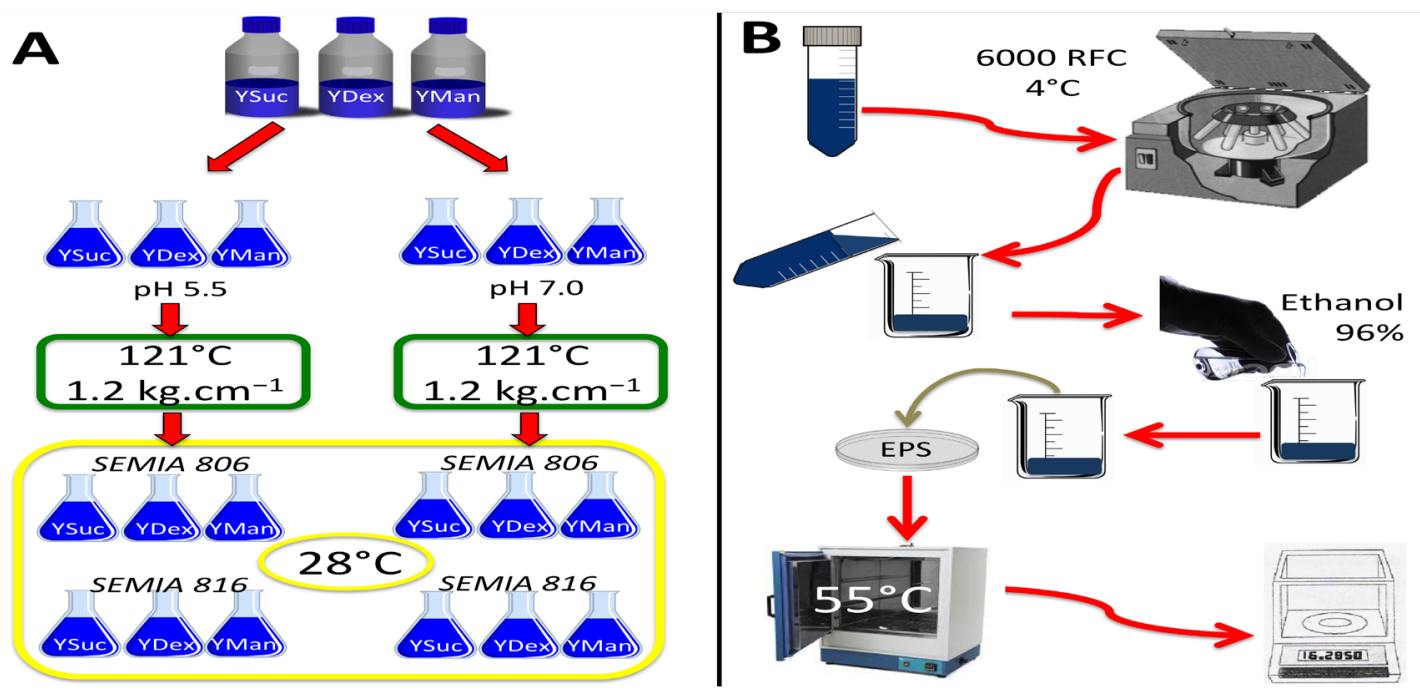

Fig1. Methodological design of the experiment. (A) Culture of M. loti strains under different $p H$ and with different carbon sources. (B) Extraction o EPSs from the cultured M. loti strains. 

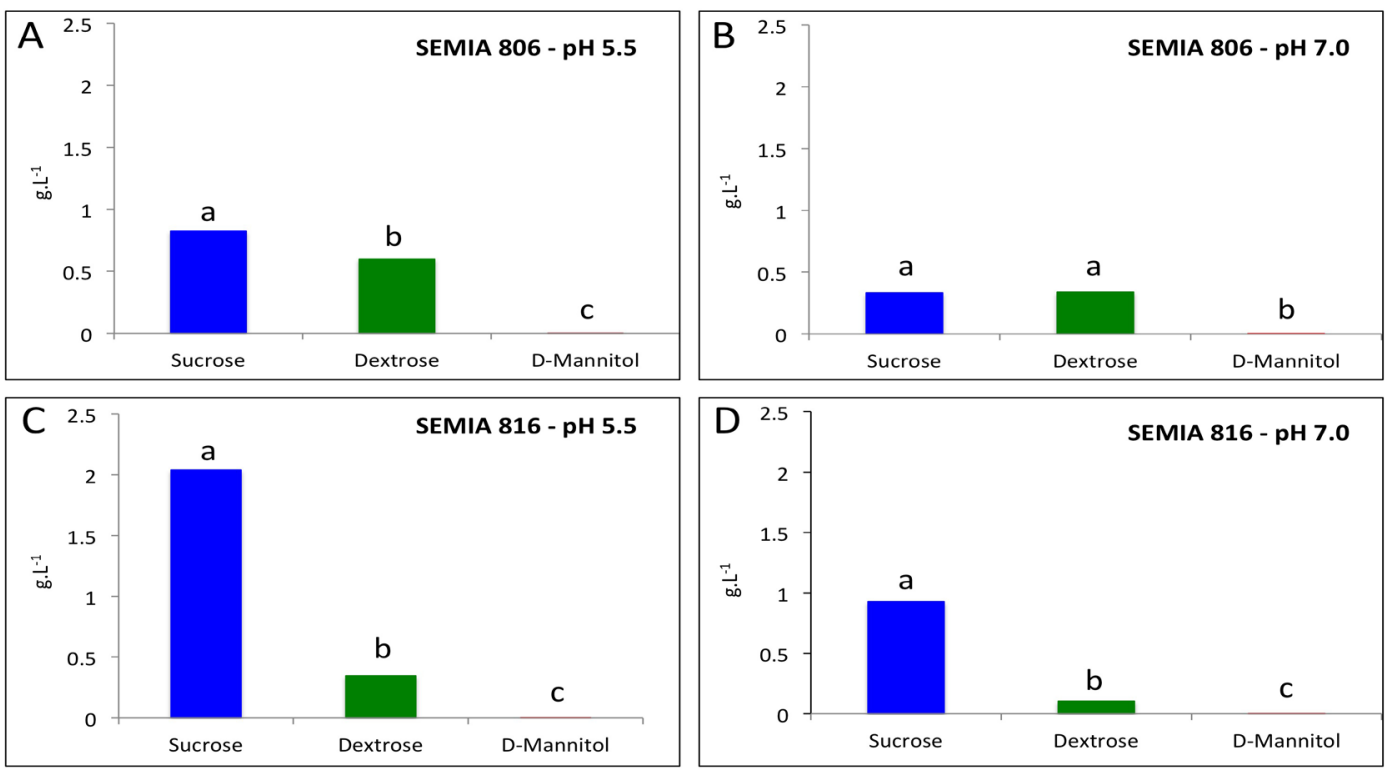

Fig2. Amount of exopolysaccharides (EPSs) produced by M. loti SEMIA806 and SEMIA816 cultured in liquid medium at $28^{\circ} \mathrm{C}$ with different carbon sources and $\mathrm{pH}$ conditions. (A) Strain SEMIA806, cultured under pH 5.5. (B). Strain SEMIA806, cultured under pH 7.0. (C) Strain SEMIA816, cultured under pH 5.5. (D) Strain SEMIA816, cultured under $p H$ 7.0. Columns with the same letter within the same treatment did not differ statistically for $\alpha=$ 5\% according to the Mann-Whitney test.

\section{Acknowledgements}

Authors thanks to FAPERGS by the PROBIC grant to JDV and to UNIPAMPA by financial support and laboratorial facilities.

\section{REFERENCES}

Ates 0. 2015. Systems biology of microbial exopolysaccharides production. Front. Bioengin. Biotechnol. 3:200.

Barberi A, Moreira FMS, Florentino LA and Rodrigues MID. 2004. Growth of Bradyrhizobium elkanii Strain BR 29 in culture media with different pH values. Ciênc. Agrotec. 28:397-405 (in Portuguese).

Bomfetti CA, Florentino LA, Guimarães AP, Cardoso PG, Guerreiro MC and Moreira FMS. 2011. Exopolysaccharides produced by the symbiotic nitrogen-fixing bacteria of Leguminosae. R. Bras. Ci. Solo. 35:657-671 (in Portuguese).

Brockwell J, Pilka A and Holliday RA. 1991. Soil pH is a major determinant of numbers of naturally occurring Rhizobium meliloti in non-cultivated soils in central New South Wales. Aust. J. Exp. Agric. 31:211-219.

Castellane TCJ, Otoboni AMMB and Lemos EGM. 2015. Characterization of exopolysaccharides by Rhizobia species. R. Bras. Ci. Solo. 39:1566-1575 (in Portuguese).

Correa OS and Barneix AJ. 1997. Celular mechanisms of pH tolerance in Rhizobium loti. World J. Microb. Biotech. 13:153-157.

Dertli E, Mayer MJ and Narbad A. 2015. Impact of exopolysaccharide layer on biofilms, adhesion and resistance to stress in Lactobacillus johnsonii FI9785. BMC Microbiol. 15:8. 
Donot F, Fontana A, Baccou JC and Schorr-Galindo S. 2012. Microbial exopolysaccharides: Main examples of synthesis, excretion, genetics and extraction. Carbohydr Polym. 87:951-62.

Fraysse N, Couderc F and Poinsot V. 2003. Surface polysaccharide involvement in establishing the rhizobium-legume symbiosis. Eur J Biochem. 270:1365-80.

Hotter GS and Scott DB. 1991. Exopolysaccharide mutants of Rhizobium loti are fully effective on a determinate nodulating host but are ineffective on an indeterminate nodulating host. J. Bact. 173:851-859.

Jarvis BDW, van Berkum P, Chen WX, Nour SM, Fernandez MP, Cleyer-Marel JC and Gillis M. 1997. Transfer of Rhizobium loti, Rhizobium huakuii, Rhizobium ciceri, Rhizobium mediterraneum, and Rhizobium tianshanense to Mesorhizobium gen. nov. Int. J. Syst. Bact. 47:895-898.

Martins AF, Vargas LK, Lisboa BB, Sampaio JAT, Araújo JHB, Turcatel AT, Diemer GD and Sá ELS. 2011. Genetic diversity, tolerance to acidity factors and symbiotic efficiency of Rhizobia of birdsffot trefoil in soils of Rio Grande do Sul. R. Bras. Ci. Solo. 35:1855-1864 (in Portuguese).

Oliveira AMR, Perez NB, Dall'agnol M, Bangel EV and Silva G. 2007. Genetic characterization of Rhizobia isolated from Lotus corniculatus L. Pesq. Agrop. Gaúcha. 13:23-27 (in Portuguese).

Sanchez-Garcia DM, Lagaron JM and Hoa SV. 2010. Effect of addition of carbon nanofibers and carbon nanotubes on properties of thermoplastic biopolymers. Compos. Sci. Technol. 70:1095-1105.

Singh RS and Saini GK. 2012. Biosynthesis of pullulan and its applications in food and pharmaceutical industry. Satyanarayana T, Johri BN and Prakash A, editors. Microorganisms in sustainable agriculture and biotechnology. New York: Springer-Verlag.

Tatsukami Y and Ueda M. 2016. Rhizobial gibberellin negatively regulates host nodule number. Sc. Reports. 6:27998.

Tiwari RP, Reeve WG and Glenn AR. 1992. Mutations conferring acid sensitivity in the acid-tolerant strains Rhizobium meiloti WSM419 and Rhizobium legominusarum biovar viciae WSM710. FEMS Microbiol. Letters. 100:107-112.

Vieira JD, Roll RJ, Rosa JVS, Castro RM and Stefenon VM. 2016. Crescimento de Rhizobium loti e Bradyrhizobium japonicum sob diferente fontes de carbono e $\mathrm{pH}$ do meio [Growth of Rhizobium loti and Bradyrhizobium japonicum under different carbon source and pH of the medium]. Annals of the VIII SIEPE; 2016 Nov 22-24; Uruguaiana, Brazil: Bagé: EdiUNIPAMPA.

Wood M, Cooper JE and Bjourson AJ. 1988. Response of Lotus rhizobia to acidity and aluminium in liquid culture and in soil. Plant and Soil. 107:227-231.

Citation: Rutilene Jacondino Roll, Jéssica Dutra Vieira, João Vitor Sales Rosa, Renata Machado Castro, Rafael Hencke Tresbach, Valdir Marcos Stefenon, "Exopolysaccharides production by Mesorhizobium loti: effect of carbon source and pH". American Research Journal of Biosciences; Volume 3, 2017; pp: 1-5

Copyright (C) 2017 Rutilene Jacondino Roll, Jéssica Dutra Vieira, João Vitor Sales Rosa, Renata Machado Castro, Rafael Hencke Tresbach, Valdir Marcos Stefenon. This is an open access article distributed under the Creative Commons Attribution License, which permits unrestricted use, distribution, and reproduction in any medium, provided the original work is properly cited. 